Anna Buschmeyer \& Dagmar Müller

\title{
Väter und Berufstätigkeit - Einführung in das Schwerpunktthemenheft
}

Das traditionelle Familienernährermodell, das für Männer eine kontinuierliche Erwerbsarbeit vorsah und Frauen auf die familiale Sorgearbeit verwies, hat in den letzten Jahrzehnten zunehmend an Boden verloren. Nicht nur hat die Arbeitsmarktbindung insbesondere von gut ausgebildeten Frauen und Müttern in den jüngeren Kohorten zugenommen (Grunow et al. 2006; Kreyenfeld/Geisler 2006). Auch wollen und sollen sich Männer, als Väter, vermehrt an der Sorgearbeit in ihren Familien beteiligen. Dass dieses Phänomen mittlerweile über die von Ulrich Beck beschriebene „,verbale Aufgeschlossenheit bei weitgehender Verhaltensstarre“ (Beck 1986, S. 169) hinausgeht, haben empirische Studien der letzten Jahre gezeigt. Väter gehen vermehrt in Elternzeit ${ }^{1}$ und bringen sich in der Folge verstärkt in die Sorgearbeit ein, vor allem, wenn sie einen Teil der Elternzeit allein für die Kinderbetreuung verantwortlich waren (Boll et al. 2014; Bünning 2015; Schober/ Zoch 2015;). Sie nehmen größeren Anteil an der Erziehung, sie verbringen insgesamt mehr Zeit mit ihren Kindern (Gauthier et al. 2004; Walper/Lien im Erscheinen) und viele halten auch nach einer Trennung bzw. Scheidung engen Kontakt (Kindler/Walper 2016). Väter sind längst nicht mehr die ,abwesenden Väter' vergangener Jahrzehnte, auch wenn ihre Praxis nicht immer mit dem Wunsch nach mehr familialem Engagement Schritt hält (Possinger 2013). So beklagen viele Väter in Deutschland, dass ihre Zeit mit Kindern nicht ausreichend sei, und wünschen sich eine Reduzierung ihrer Arbeitszeit. Gleichwohl arbeiten die meisten Väter Vollzeit und durchschnittlich sogar eine Stunde länger als Männer ohne minderjährige Kinder im Haushalt (Bujard/Schwebel 2015). Unabhängig vom Alter der Kinder sind nur fünf bis sechs Prozent der Väter teilzeiterwerbstätig, während es bei den Müttern durchschnittlich 70 Prozent sind (Hobler et al. 2017). Entsprechend bleiben Väter meist die Hauptverdiener in den Familien, nicht zuletzt deshalb, weil ihre Einkommen die von Frauen in vergleichbaren Positionen und Berufen immer noch übersteigen (Boll et al. 2016).

1 Die Väterbeteiligung beim 2007 eingeführten Elterngeld liegt aktuell bei 36\% (Statistisches Bundesamt 2017).

Zeitschrift für Familienforschung, 29. Jahrg., 2017, Heft 1 - Journal of Family Research

https://doi.org/10.3224/zff.v29i1.01 
Die offenkundige Diskrepanz zwischen dem Wunsch nach mehr familialem Engagement bei gleichbleibendem Erwerbsumfang bedeutet in der Konsequenz, dass sich Väter in vergleichbaren Dilemmata wiederfinden, die für Mütter schon lange - wenn auch mit unterschiedlichen Vorzeichen - Realität waren und sind. Während es für Mütter nach wie vor schwierig ist, die Anforderungen familialer Sorgearbeit mit Erwerbsarbeit zu vereinen, scheint es für Väter umgekehrt eine Herausforderung darzustellen, die Ansprüche, die von Erwerbsarbeitsseite gestellt werden, mit den Bedürfnissen eines Familienlebens zu vereinbaren. Im Zuge dieser Veränderungen hat das wissenschaftliche Interesse an Vätern stark zugenommen. Noch vor gut zehn Jahren konstatierten Angelika Tölke und Karsten Hank die Männer als das ,,vernachlässigte“ Geschlecht in der Familienforschung" (Tölke/Hank 2005). Heute scheint Männer- und Väterforschung aus der Familienforschung, ebenso wie aus der Geschlechterforschung, kaum noch wegzudenken, wie zahlreiche Veröffentlichungen der letzten Jahre belegen (z.B. Adler/Lenz 2017; Behnke 2012; Buschmeyer 2008; Eydal/Rostgard 2015; Johansson 2011; Kassner 2008; Klinger 2015; Kvande/Brandth 2017; Lengersdorf/Meuser 2016; Li et al. 2015; Meuser 2014; Peukert 2015; Possinger 2013; Prietl 2016; Richter 2012; Ruspini/Crespi 2016; Walter/Eickhorst 2011). Studien, die Väter und Berufstätigkeit ist den Mittelpunkt stellen, sind allerdings weiterhin deutlich seltener als solche, die Mütter und Berufstätigkeit untersuchen (Kreyenfeld 2015), da Männlichkeit nicht automatisch mit (potentieller) Väterlichkeit gleichgesetzt wird. Auch wird bei Vätern nicht unbedingt erwartet, dass ihre berufliche Verfügbarkeit durch die Sorge für Kinder beeinflusst wird - während dies bei Frauen bzw. Müttern in der Regel vorausgesetzt wird. Andererseits erscheint die schwierige Vereinbarkeit von Familie und Beruf als ein wesentlicher Hemmschuh für ein verstärktes väterliches Engagement.

Die Beschäftigung mit Vätern und Berufstätigkeit verspricht interessante wissenschaftliche Erkenntnisse, weil sie einen Testfall für gängige theoretische Erklärungen des Erwerbsverhaltens, insbesondere im Haushaltskontext, darstellt und die Frage nach einer Neukonfigurierung von Männlichkeit und Väterlichkeit aufwirft (Lengersdorf/Meuser 2016). Sie ist gleichzeitig politisch und praktisch relevant, weil sie auf grundlegende Fragen nach Möglichkeiten und Bedingungen einer gleichmäßigeren Aufteilung von Erwerbs- und Sorgearbeit zwischen Männern und Frauen verweist. Wie jüngst die OECDStudie „Dare to Share“ ergab, besteht in Deutschland diesbezüglich weiter Handlungsbedarf. So ist die Erwerbsarbeitszeitdifferenz zwischen zusammenlebenden (Ehe-)Partnern in Deutschland größer als in anderen westeuropäischen Staaten und den USA und der Einkommensanteil der Frauen am Haushaltseinkommen entsprechend kleiner (OECD 2017: 25). Das bedeutet, dass Frauen nach wie vor die Hauptlast der unbezahlten Sorgearbeit tragen, mit entsprechend negativen Konsequenzen für ihr Lebenserwerbseinkommen.

$\mathrm{Ob}$ und unter welchen Bedingungen Väter bereit sind, ihr berufliches Engagement zu Gunsten der Übernahme von Sorgeverantwortung zu reduzieren, ist für den deutschen Kontext erst ansatzweise erforscht. Längsschnittstudien wie die von Pollmann-Schult (2008) deuten darauf hin, dass sich im Übergang zur Elternschaft zwar der präferierte, kaum aber der faktische Erwerbsarbeitsumfang von Männern verändert. Damit erscheint das Erwerbsarbeitsverhalten von Männern vergleichsweise invariant oder eher dem bekannten ,Traditionalisierungseffekt ${ }^{\star}$ nach einer Familiengründung zu folgen (Kühhirt 
2012). Auch im Kohortenvergleich zeigen sich die Erwerbsverläufe von Männern wenig beeinflusst vom Vorhandensein von Kindern (Simonson et al. 2014). Erklärungsansätze beziehen sich auf Geschlechternormen und partnerschaftliche Aushandlungsprozesse, Ressourcenunterschiede im Haushaltskontext, arbeitsmarktliche und betriebliche Rahmenbedingungen sowie wohlfahrtsstaatliche Politiken, die die geschlechtliche Arbeitsteilung in Familien beeinflussen (Kreyenfeld 2015; Schiefer/Bujard 2012). Als ein Hauptproblem gelten hohe berufliche Verfügbarkeitserwartungen, die mit privater Sorgearbeit kulturell kaum kompatibel sind (Possinger 2013; Williams et al. 2013). Gemäß der ,idealworker norm" (Williams 2000) hat sich ein Arbeitnehmer zeitlich und kognitiv-emotional ganz der Arbeit zu widmen, während sich väterliche Fürsorge im bestmöglichen Erfüllen der Ernährerrolle manifestiert. Väter, die diesen kulturellen Modellen nicht folgen und ihre Erwerbsarbeitszeit reduzieren, unterliegen einem „flexibility stigma“ (Williams et al. 2013) und müssen mit beruflichen Nachteilen wie Lohneinbußen und geringeren Aufstiegschancen rechnen (Bünning 2016; Coltrane et al. 2013; Rege/Solli 2013). Das erklärt, warum viele Väter - und auch Mütter bzw. Personen mit Sorgeverpflichtungen - Elternzeiten und flexible Arbeitszeitmodelle trotz formalem Angebot nicht in Anspruch nehmen.

Ausgehend von diesen aktuellen Diskussionen versammelt das Schwerpunktthemenheft empirisch fundierte Beiträge, die die komplexen Wechselbeziehungen von Vaterschaft und Berufstätigkeit beleuchten: Welche Folgen hat (werdende) Vaterschaft für die Berufstätigkeit? Wie wird umgekehrt das Engagement der Väter in der Familie durch Berufstätigkeit bzw. Erwerbsarbeit beeinflusst? Welche Bedingungen hemmen oder fördern väterliches Engagement in Familie und Beruf? Wie werden zudem im öffentlichen Diskurs, in Arbeitsorganisationen und in Familien Vorstellungen von Männlichkeit, Väterlichkeit und väterlicher Berufstätigkeit (re-)konstruiert?

Der Beitrag von Claudia Zerle-Elsäßer und Xuan Li befasst sich mit der Rolle von Vätern im Familienalltag und fragt hierbei nach den Determinanten für (überdurchschnittliches) väterliches Engagement. Auf Basis des DJI-Surveys AID:A II bilden die Autorinnen dazu einen Index ,aktiver Vaterschaft', der den zeitlichen Umfang und die in Relation zu Müttern anteilige Beteiligung der Väter an kindbezogenen Aktivitäten umfasst. Ihre Analysen beziehen Zielkinder im Alter bis unter neun Jahren und deren beide Elternteile ein. Im Anschluss an die familienwissenschaftliche Väterforschung der letzten Jahre untersuchen die Autorinnen erstens individuelle Determinanten für väterliches Engagement, vor allem die Genderkonzepte der befragten Väter. Zweitens betrachten sie partnerschaftsbezogene Aspekte wie die Partnerschaftszufriedenheit und die Zusammenarbeit in der Erziehung (Coparenting), und drittens ökonomische bzw. erwerbsbezogene Faktoren. Diese erweisen sich in den Analysen als stärkste Prädiktoren ,aktiver Vaterschaft": Lange Wochenarbeitszeiten der Väter, insbesondere Überstunden, sind demnach abträglich für väterliches Engagement, während sich die Wahrscheinlichkeit, als Vater aktiv zu sein, mit zunehmender Erwerbsarbeitszeit der Mutter erhöht. Interessanterweise wird dieser Effekt erst bei einer Zahl von 36 bis 40 Wochenstunden, also einer Vollzeiterwerbstätigkeit der Mutter signifikant. Entsprechend ist das väterliche Engagement umso höher, je mehr die Frau zum Haushaltseinkommen beiträgt. Als weitere relevante Einflussgrößen machen die Autorinnen den Zusammenhalt der Eltern in der Erziehung (Coparenting) und die Genderkonzepte insbesondere der Mütter aus. 
Auch Lena Hipp, Friederike Molitor, Janina Leschke und Sonja Bekker wenden sich dem Thema Erwerbsarbeitszeit von Vätern zu. In einem Vergleich zwischen Deutschland, Schweden, Irland und den Niederlanden untersuchen sie, unter welchen Bedingungen Väter in Paarbeziehungen Teilzeit arbeiten. Dabei stützen sie sich auf Individualdaten aus der Europäischen Arbeitskräfteerhebung (EU AKE) aus dem Jahr 2014. Ihre Ergebnisse zeigen, dass, entgegen haushaltsökonomischen und verhandlungstheoretischen Annahmen, berufliche Statusunterschiede auf Paarebene in keinem der Länder mit unterschiedlichen Teilzeitwahrscheinlichkeiten von Vätern einhergehen. Väter mit ähnlichem oder niedrigerem beruflichen Status als ihre Partnerinnen arbeiten in keinem der Länder mit einer höheren Wahrscheinlichkeit in Teilzeit als Väter, die einen Beruf von höherem beruflichen Status ausüben. Unabhängig davon arbeiten Väter in Deutschland mit höherer Wahrscheinlichkeit in Teilzeit, wenn sie kleine Kinder oder drei und mehr Kinder zu versorgen haben. In Irland, Schweden und den Niederlanden zeigen sich andere Zusammenhänge, was die Autorinnen auf unterschiedliche wohlfahrtsstaatliche Rahmenbedingungen zurückführen. Insgesamt schlussfolgern die Autorinnen, dass für Väter andere Faktoren für Teilzeitarbeit relevant sind als für Mütter und dass die Logik der Spezialisierung und Nutzenmaximierung aufgrund unterschiedlicher Einkommenspotentiale in Paarbeziehungen in diesem Fall nicht greift. Eher scheinen normative Geschlechtervorstellungen und normative Erwartungen eine Rolle zu spielen.

Janine Bernhardt und Mareike Bünning wenden sich ebenfalls der Erwerbsarbeitszeit von Vätern zu und lenken den Blick von der Paarebene auf die betriebliche Ebene. Anhand einer Zusatzerhebung zum AID:A-Survey des DJI untersuchen sie, welche betriebliche Rahmenbedingungen kürzere Arbeitszeiten von Vätern begünstigen. Dabei gehen sie davon aus, dass bei Vätern die Inanspruchnahme von Rechtsansprüchen und betrieblichen Vereinbarkeitsangeboten in hohem Maße von organisationskulturellen Faktoren abhängt, weshalb auch zwischen vertraglichen und tatsächlichen Arbeitszeiten unterschieden werden muss. In ihrer Untersuchung nehmen sie vier betriebskulturelle Aspekte in den Blick: Erstens, wie stark Väter im Unternehmen Verfügbarkeitserwartungen wahrnehmen (,idealworker norm“); zweitens, inwiefern es nach Einschätzung der Väter transparente betriebliche Regelungen gibt, an denen sie sich orientieren können; drittens, inwieweit sie sich bei der Vereinbarkeit von Familie und Beruf von ihren direkten Vorgesetzten unterstützt fühlen; und viertens, wie leicht sie im Kollegenkreis eine Vertretung finden können. Zusätzlich vergleichen die Autorinnen betriebsstrukturelle Merkmale wie die Betriebsgröße, die Branche, die Zugehörigkeit des Unternehmens zum öffentlichen Dienst und ob ein Betriebs- oder Personalrat vorhanden ist. Die Ergebnisse zeigen einen robusten Zusammenhang zwischen dem Ausmaß an formellen, allgemeingültigen und transparenten Regelungen im Betrieb und kürzeren Arbeitszeiten von Vätern. Entscheidend ist dabei, dass die Väter subjektiv das Gefühl haben, dass die Unternehmensleitung sie bei ihren Vereinbarkeitsbemühungen unterstützt. Betriebskulturelle Faktoren sind Bernhardt und Bünning zufolge vor allem für die tatsächlichen Arbeitszeiten (z.B. Vermeidung von Mehrarbeit), weniger für die vertraglichen Arbeitszeiten relevant. Strukturelle Faktoren wie Branche oder Betriebsgröße spielen demgegenüber eine untergeordnete Rolle, was sich dahingehend interpretieren lässt, dass väterfreundliche Arbeitszeitgestaltungen prinzipiell in allen Betrieben möglich sind und es vor allem auf den Gestaltungswillen der Unternehmensleitungen ankommt. 
Der Bedeutung von Betriebs- bzw. Organisationskulturen gehen Annette von Alemann, Sandra Beaufä̈s und Mechtild Oechsle in ihrer qualitativen Untersuchung weiter auf den Grund. Anhand von zwei Unternehmensfallstudien, die im Rahmen des Sonderforschungsbereichs (SFB) 882 „Von Heterogenitäten zu Ungleichheiten“ an der Universität Bielefeld entstanden sind, zeigen sie auf, wie Organisationskulturen Ansprüche und Handeln von Vätern beeinflussen. Der Fokus liegt dabei auf den informellen Regeln im Unternehmensalltag (,hidden rules“), die oftmals in Widerspruch zu offiziellen Unternehmenspolitiken stehen und es Vätern erschweren, ein Anspruchsbewusstsein hinsichtlich der Vereinbarkeit zu entwickeln, zu formulieren und durchzusetzen. Die beiden Fallbeispiele, ein öffentlich-rechtliches Versicherungsunternehmen und ein privatwirtschaftliches Unternehmen im Konsumgüterhandel, unterscheiden sich zum Teil beträchtlich in ihren Führungs-, Arbeitszeit- und Geschlechterkulturen. Beiden gemeinsam ist, dass sie als familienfreundliche Betriebe ausgezeichnet sind. Dennoch können viele Väter ihre Vereinbarkeitswünsche nicht realisieren. Die Autorinnen zeigen detailliert auf, welche verborgenen Regeln (z.B. männlich konnotierte Karrierenormen) und sozialen Sanktionen (z.B. unter Kolleg(inn)en) dazu führen, dass Väter die bestehenden Angebote nicht nutzen. Als ein Haupthindernis machen sie aus, dass sich Väter nicht berechtigt fühlen, die Angebote zu nutzen, da diese vorrangig Mütter adressieren und eine ausgesprochene Erlaubnis zur Nutzung der Angebote durch Vorgesetzte fehlt. Zudem werden Abweichungen von den organisationsinternen Normen negativ sanktioniert. Dadurch, so die Autorinnen, behindern die Organisationskulturen die Ausprägung eines Anspruchsbewusstseins bei den Vätern.

Almut Peukert befasst sich mit der Frage, inwieweit ,involvierte' Väterlichkeit zu neuen Konstruktionen und Praktiken von Männlichkeit führt, die die traditionelle Erwerbszentrierung hegemonialer Männlichkeit infrage stellen. Gestützt auf qualitative Einzel- und Paarinterviews untersucht sie paarinterne Aushandlungen zur Erwerbs- und Sorgearbeit und zeigt dabei auf, dass väterliches Engagement nicht automatisch zu einem Aufbrechen der Geschlechterordnung führt. Anhand der Dimensionen Berufs-/Karrierekonzept, finanzielle Versorgung der Familie und Elternzeitarrangement rekonstruiert sie drei unterschiedliche Formen der Re/Produktion erwerbszentrierter hegemonialer Männlichkeit: (1) fragile, (2) herausgeforderte Re/Produktion und (3) episodisches Aussetzen der Re/Produktion. Die fragile Re/Produktion hegemonialer Männlichkeit zeichnet sich durch die Hierarchisierung und Priorisierung von Erwerbs- über Familienarbeit aus, die auf diskursiver Ebene zunehmend legitimationsbedürftig wird. Der Wunsch, sich als Vater stärker zu engagieren, wird als unter den gegebenen Bedingungen der Erwerbsarbeit nicht umsetzbar dargestellt. Der zweite Typus der herausgeforderten Re/Produktion ist gekennzeichnet von einer ambivalenten Gleichzeitigkeit der Infragestellung und Bekräftigung hegemonialer Männlichkeit. Das Paar befindet sich in ständiger Aushandlung darüber, wer welche Aufgaben übernimmt und übernehmen darf und in wieweit diese Aufgabenteilung sich an traditionellen Geschlechternormen orientieren soll. Das episodische Aussetzen der Re/Produktion (hegemonialer) Männlichkeit ist charakterisiert durch eine nicht-hierarchisierende und -priorisierende, geschlechtsindifferente Organisation von Erwerbs- und Familienarbeit innerhalb des Paares. Dies deutet Peukert als situatives „Vergessen des Geschlechts“" (Hirschauer 2001), das über hegemoniale Männlichkeit hinausweist. 
Der Beitrag von Kathrin Peltz, Luisa Streckenbach, Dagmar Müller, Johanna Possinger und Barbara Thiessen beschließt den Kreis der Beiträge in diesem Schwerpunktthemenheft. Ausgehend von der bemerkenswert hohen Nutzung der ,Vätermonate' beim Elterngeld in Bayern sowie den gleichzeitig zu beobachtenden großen regionalen Unterschieden der Inanspruchnahme fragen die Autorinnen danach, welche Bedeutung die Erwerbsarbeit für den Elterngeldbezug von Vätern hat. Auf der Grundlage aggregierter Regionaldaten werden zunächst erwerbsbezogene Kontextfaktoren für den Elterngeldbezug in den bayerischen Regionen untersucht. Dabei zeigt sich, dass insbesondere eine geringe Arbeitslosigkeit, das Arbeitsplatzangebot für hoch qualifizierte Beschäftigte und eine ausgewogene Erwerbsbeteiligung von Frauen und Männern mit einer höheren Väterbeteiligung am Elterngeld einhergehen. Das Einkommensniveau steht wider Erwarten in keinem Zusammenhang, sodass die hohe Väterbeteiligung nicht auf das in Bayern höhere Wohlstandsniveau zurückgeführt werden kann. In einem weiteren Schritt werden auf der Grundlage problemzentrierter Interviews mit Vätern und Paaren individuelle Begründungen für den Elterngeldbezug des Vaters rekonstruiert. Deutlich wird unter anderem, dass Erwerbsarbeit für Väter mit eigenen Zeitlogiken, Rhythmisierungen und vor allem auch emotionalen Bezügen verknüpft ist, die es ihnen erschweren, sich über die zwei Partnermonate hinaus auf eine Elternzeit einzulassen.

Abschließend möchten wir an dieser Stelle allen Autorinnen, den anonymen Gutachterinnen und Gutachtern, den Herausgeberinnen und Herausgebern sowie der Redaktion der Zeitschrift für Familienforschung, namentlich Dr. Kurt Bierschock, für das Zustandekommen des Schwerpunktthemenhefts und die hilfreiche Unterstützung danken.

\section{Literatur}

Adler, M. \& Lenz, K. (Hrsg.) (2017). Father involvement in the early years. An international comparison of policy and practice. Bristol: Policy Press.

Beck, U. (1986). Risikogesellschaft. Auf dem Weg in eine andere Moderne. Frankfurt am Main: Suhrkamp.

Behnke, C. (2012). Partnerschaftliche Arrangements und väterliche Praxis in Ost- und Westdeutschland. Paare erzählen. Opladen: Verlag Barbara Budrich.

Boll, C., Leppin, J. \& Reich, N. (2014). Paternal childcare and parental leave policies: evidence from industrialized countries. Review of Economics of the Household, 12, 1, S.129-158.

Boll, C., Rossen, A. \& Wolf, A. (2016). The EU gender earnings gap: Job segregation and working time as driving factors. Nürnberg: Institut für Arbeitsmarkt- und Berufsforschung (IAB-Discussion Paper Nr. 36/2016).

Bünning, M. (2015): What happens after the 'daddy months'? Fathers' involvement in paid work, childcare, and housework after taking parental leave in Germany. European Sociological Review, 31, 6, S. 738-748.

Bünning, M. (2016). Die Vereinbarkeitsfrage für Männer: Welche Auswirkungen haben Elternzeiten und Teilzeitarbeit auf die Stundenlöhne von Vätern? Kölner Zeitschrift für Soziologie und Sozialpsychologie, 68, 4, S. 597-618.

Bujard, M. \& Schwebel, L. (2015). Väter zwischen Wunsch und Realität. Neue Vereinbarkeitsprobleme von Familie und Beruf bei Männern. Gesellschaft, Wirtschaft, Politik (GWP), 64, 2, S. 211-224.

Buschmeyer, A. (2008). Männlichkeitskonstruktionen Teilzeit arbeitender Väter. In: Baur, N. \& Luedtke, J. (Hrsg.), Die soziale Konstruktion von Männlichkeit. Hegemoniale und marginalisierte Männlichkeiten in Deutschland. Opladen: Verlag Barbara Budrich, S. 123-140. 
Coltrane, S., Miller, E. C., DeHaan, T. \& Stewart, L. (2013). Fathers and the flexibility stigma. Journal of Social Issues, 69, 2, S. 279-302.

Eydal, B. G. \& Rostgard, T. (Hrsg.) (2015). Fatherhood in the Nordic welfare states: Comparing care policies and practice. Bristol: Policy Press.

Gauthier, A. H., Smeeding, T. M. \& Furstenberg jr., F. F. (2014). Are parents investing less time in children? Trends in selected industrialized countries. Population and Development Review, 30, 4, S. 647-672.

Grunow, D., Hofmeister, H. \& Buchholz, S. (2006): Late 20th century persistence and decline of the female homemaker in Germany and the United States. International Sociology, 21, 1, S. 101-132.

Hirschauer, S. (2001). Das Vergessen des Geschlechts. Zur Praxeologie einer Kategorie sozialer Ordnung. Kölner Zeitschrift für Soziologie und Sozialpsychologie, 53, Sonderheft 41, S. 208-235.

Hobler, D., Pfahl, S. \& Horvath, S. (2017). Teilzeitquoten nach Elternschaft und Alter des jüngsten Kindes 2014. WSIGenderDatenPortal, https://www.boeckler.de/51974.htm [Stand: 2017-03-10].

Johansson, T. (2011). Fatherhood in transition: Paternity leave and changing masculinities. Journal of Family Communication, 11, 3, S.165-180.

Kassner, K. (2008). Männlichkeitskonstruktionen von ,neuen Vätern‘. In: Baur, N. \& Luedtke, J. (Hrsg.), Die soziale Konstruktion von Männlichkeit. Hegemoniale und marginalisierte Männlichkeiten in Deutschland. Opladen: Verlag Barbara Budrich, S. 141-163.

Kindler, H. \& Walper, S. (2016): Das Wechselmodell im Kontext elterlicher Konflikte. Neue Zeitschrift für Familienrecht, 3, 18, S. 820-824.

Klinger, L.-M. (2015). Der Papa, der macht alles, was sonst keiner gerne tut? Deutungsmuster von Vaterschaft aus der Perspektive von Fachkräften im Jugendamt. In: Seehaus, R., Rose, L. \& Günther, M. (Hrsg.), Vater, Mutter, Kind? - Geschlechterpraxen in der Elternschaft. Leverkusen: Verlag Barbara Budrich (Geschlechterforschung für die Praxis 3), S. 223-241 (1. Auflage).

Kreyenfeld, M. (2015). Maternal and paternal employment across the life course In: Scott, R. A., Kosslyn, S. M. (Hrsg.), Emerging trends in the social and behavioral sciences: An interdisciplinary, searchable, and linkable resource. Hoboken: Wiley, S. 1-15.

Kreyenfeld, M. \& Geisler, E. (2006). Müttererwerbstätigkeit in Ost- und Westdeutschland. Zeitschrift für Familienforschung, 18, 3, S. 333-360.

Kühhirt, M. (2012). Childbirth and the long-term division of labour within couples: How do substitution, bargaining power, and norms affect parents' time allocation in West Germany? European Sociological Review, 28, 5, S. 565-582.

Kvande, E. \& Brandth, B. (2017). Fathers on leave alone in Norway: Changes and continuities. In: O'Brien, M. \& Wall, K. (Hrsg.), Comparative perspectives on work-life balance and gender equality. Fathers on leave alone. London: Springer Open (Life Course Research and Social Policies 6), S. 29-44.

Lengersdorf, D. \& Meuser, M. (2016). Involved fatherhood: Source of new gender conflicts? In: Crespi, I. \& Ruspini, E. (Hrsg.), Balancing work and family in a changing society. The fathers' perspective. Houndmills, Basingstoke, Hampshire \& New York: Palgrave Macmillan (Global masculinities), S. 149-161.

Li, X., Zerle-Elsäßer, C., Entleitner-Phleps, C. \& Schier, M. (2015). Väter 2015: Wie aktiv sind sie, wie geht es ihnen und was brauchen sie? München: Deutsches Jugendinstitut.

Meuser, M. (2014). Care und Männlichkeit in modernen Gesellschaften - Grundlegende Überlegungen illustriert am Beispiel involvierter Vaterschaft. In: Aulenbacher, B., Riegraf, B. \& Theobald, H. (Hrsg.), Sorge: Arbeit, Verhältnisse, Regime. Baden-Baden: Nomos (Soziale Welt: Sonderband 20), S. 159-174 (1. Auflage).

OECD (2017). Dare to Share - Deutschlands Weg zur Partnerschaftlichkeit in Familie und Beruf. Paris: OECD Publishing.

Peukert, A. (2015). Aushandlungen von Paaren zur Elternzeit. Arbeitsteilung unter neuen Vorzeichen? Wiesbaden: Springer VS (Geschlecht und Gesellschaft, Band 61).

Pollmann-Schult, M. (2008). Familiengründung und gewünschter Erwerbsumfang von Männern - Eine Längsschnittanalyse für die alten Bundesländer. Zeitschrift für Soziologie, 37, 6, S. 498-515. 
Possinger, J. (2013). Vaterschaft im Spannungsfeld von Erwerbs- und Familienleben. „Neuen Vätern“ auf der Spur. Wiesbaden: Springer VS (Research).

Prietl, B. (2016). Ambivalente Männlichkeitskonstruktionen von Vätern zwischen Erwerbsarbeit und Fürsorgearbeit. Gender - Zeitschrift für Geschlecht, Kultur und Gesellschaft, 8, 1, S. 124-139.

Rege, M. \& Solli, I. F. (2013). The impact of paternity leave on fathers' future earnings. Demography, 50, 6, S. 2255-2277.

Richter, R. (2012). Väter in Elternzeit - Umsetzungen und Strategien zwischen Familie und Beruf. Paderborn: Universität Paderborn (Dissertation). http://digital.ub.uni-paderborn.de/hs/id/451072. [Stand: 2017-02-13].

Ruspini, E. \& Crespi, I. (Hrsg.) (2016). Balancing work and family in a changing society: The fathers' perspective. New York: Palgrave Macmillan.

Schiefer, K. \& Bujard, M. (2012). „Papa arbeitet viel“: Lange Arbeitszeit von deutschen Vätern und mögliche Ursachen. Bevölkerungsforschung Aktuell, 6/2012, S. 10-16.

Schober, P. S. \& Zoch, G. (2015). Kürzere Elternzeit von Müttern: Gleichmäßigere Aufteilung der Familienarbeit? DIW-Wochenbericht, 82, 50, S. 1190-1196.

Simonson, J., Romeu Gordo, L. \& Kelle, N. (2014). Parenthood and subsequent employment: Changes in the labor participation of fathers across cohorts as compared to mothers. Fathering, 12, 3, S. 320336.

Tölke, A. \& Hank, K. (Hrsg.). (2005). Männer - das „,vernachlässigte“ Geschlecht in der Familienforschung. Zeitschrift für Familienforschung, Sonderheft 4. Wiesbaden: VS Verlag für Sozialwissenschaften.

Statistisches Bundesamt (2017). Väterbeteiligung beim Elterngeld steigt weiter an. Pressemitteilung Nr. 054 vom 15.02.2017. Wiesbaden: Statistisches Bundesamt.

Walper, S. \& Lien, S.-C. (im Erscheinen). Aktive Vaterschaft im Kontext unterschiedlicher Familienphasen und Erwerbskonstellationen. In: Statistisches Bundesamt (Hrsg.), Ergebnisse der Zeitverwendungserhebung 2012/13. Wiesbaden: Statistisches Bundesamt.

Walter, H. \& Eickhorst, A. (Hrsg.) (2011). Das Väter-Handbuch. Theorie, Forschung, Praxis. Gießen: Psychosozial.

Williams, J. (2001). Unbending gender: Why family and work conflict and what to do about it. Oxford: Oxford University Press.

Williams, J. C., Blair-Loy, M. \& Berdahl, J. L. (2013). Cultural schemas, social class, and the flexibility stigma. Journal of Social Issues, 69, 2, S. 209-234.

Anschriften der Autorinnen/Addresses of the authors:

Dr. Anna Buschmeyer

Dagmar Müller, Diplom-Sozialwissenschaftlerin

Deutsches Jugendinstitut e.V. (DJI)

Nockherstr. 2

81541 München

Deutschland/Germany

E-Mail/Email: buschmeyer@dji.de dmueller@dji.de 